\title{
A study of noninvasive fractional flow reserve derived from a simplified method based on coronary computed tomography angiography in suspected coronary artery disease
}

Changzheng Shi ${ }^{1 \dagger}$, Dong Zhang ${ }^{1 \dagger},{\text { Kunlin } \mathrm{CaO}^{2 \dagger}, \text { Tao Zhang }^{1} \text {, Liangping Luo }{ }^{1 *} \text {, Xin } \mathrm{Liu}^{3^{*}} \text { and Heye Zhang }}^{3}$

\section{*Correspondence:}

tluolp@jnu.edu.cn;

liu.xin@siat.ac.cn

${ }^{\dagger}$ Changzheng Shi, Dong

Zhang and Kunlin Cao

contributed equally to this

work

1 Medical Imaging Center,

The First Affiliated Hospital,

Jinan University, 613

Huangpu W Ave, Tianhe

District, Guangzhou 510630,

Guangdong Province, China

${ }^{3}$ Shenzhen Institutes

of Advanced Technology,

Chinese Academy

of Sciences, 1068 Xueyuan

Ave. Xili University

Town, Nanshan District,

Shenzhen 518055,

Guangdong Province, China

Full list of author information is available at the end of the article

\begin{abstract}
Background: The invasive fractional flow reserve has been considered the gold standard for identifying ischaemia-related stenosis in patients with suspected coronary artery disease. Determining non-invasive FFR based on coronary computed tomographic angiography datasets using computational fluid dynamics tends to be a demanding process. Therefore, the diagnostic performance of a simplified method for the calculation of FFR $\mathrm{FTA}_{\mathrm{A}}$ requires further evaluation.

Objectives: The aim of this study was to investigate the diagnostic performance of FFR ${ }_{C T A}$ calculated based on a simplified method by referring to the invasive FFR in patient-specific coronary arteries and clinical decision-making.
\end{abstract}

Methods: Twenty-nine subjects included in this study underwent CCTA before undergoing clinically indicated invasive coronary angiography for suspected coronary artery disease. Pulsatile flow simulation and a novel boundary condition were used to obtain FFR $_{\text {CTA }}$ based on the CCTA datasets. The Pearson correlation, Bland-Altman plots and the diagnostic performance of FFR $\mathrm{CTA}_{\text {and }}$ and $\mathrm{CCT}$ stenosis were analyzed by comparison to the invasive FFR reference standard. Ischaemia was defined as an FFR or FFR $\leq 0.80$, and anatomically obstructive CAD was defined as a CCTA stenosis $>50 \%$.

Results: $F F R_{C T A}$ and invasive FFR were well correlated $(r=0.742, P=0.001)$. Slight systematic underestimation was found in $\mathrm{FFR}_{\mathrm{CTA}}$ (mean difference 0.03 , standard deviation $0.05, P=0.001$ ). The area under the receiver-operating characteristic curve was 0.93 for FFR CTA $_{\text {A }}$ and 0.75 for CCTA on a per-vessel basis. Per-patient accuracy, sensitivity and specificity were 79.3, 93.7 and 61.5\%, respectively, for FFR CTA $_{\text {and }} 62.1,87.5$ and $30.7 \%$, respectively, for CCTA. Per-vessel accuracy, sensitivity and specificity were 80.6, 94.1 and $68.4 \%$, respectively, for FFR $\mathrm{CTA}_{\mathrm{A}}$ and $61.6,88.2$ and $36.8 \%$, respectively, for CCTA.

Conclusions: FFR $_{C T A}$ derived from pulsatile simulation with a simplified novel boundary condition was in good agreement with invasive FFR and showed better diagnostic performance compared to CCTA, suggesting that the simplified method has the potential to be an alternative and accurate way to assess the haemodynamic characteristics for coronary stenosis.

Keywords: CCTA, FFR $_{C T A}$, FFR, CFD 


\section{Background}

Coronary artery disease (CAD), the most common type of heart disease, has become the leading cause of death among Chinese adults [1]. The presence of myocardial ischaemia is the most important risk factor for an adverse outcome, and the revascularization of ischaemia-related stenotic coronary lesions can improve patients' functional status in the clinic [2]. Coronary revascularization is often performed based on semi-quantitative measures of stenosis during invasive coronary angiography (ICA) [3]. However, the relationship between coronary stenosis severity and myocardial ischaemia is unreliable. In lesions with stenosis $<50,50-70 \%$ and $>70 \%$, only 9,18 and $57 \%$ of lesions are ischaemia causing [4]. This suggests that basing clinical treatment decisions on stenosis severity alone would result in unnecessary procedures; physiological information may be more important.

At present, the fractional flow reserve (FFR) has been regarded as the gold standard in the assessment of haemodynamic characteristics for coronary stenosis [5], and it is recommended for clinical treatment decision making before coronary revascularization [6]. In the FAME (fractional flow reserve versus angiography for multivessel evaluation) trial, FFR-guided revascularization (revascularization for lesions with FFR $\leq 0.80$ ) led to an approximately $28 \%$ lower rate of major adverse cardiac events compared with an angiography-guided strategy [7]. However, FFR is applied to guiding management in less than $10 \%$ of percutaneous coronary intervention (PCI) cases [8]. The high cost of the coronary pressure wire and the invasive medical operation may hinder the application of FFR measurements in the clinic $[9,10]$.

Coronary computed tomographic angiography (CCTA) has emerged as a non-invasive method to visualize CAD and assess anatomic stenosis severity [11-13]. In recent years, the advances in computational fluid dynamics (CFD) have made it possible to simulate and calculate the coronary flow and pressure from anatomic imaging data [14]. Based on the reconstructions from CCTA images, FFR without additional medications could be calculated. Integrating anatomic and functional information, non-invasive FFR derived from CTA $\left(\mathrm{FFR}_{\mathrm{CTA}}\right)$ may be an available and cost-effective method to identify individuals who will or will not benefit from coronary revascularization.

Several randomized trials have shown that the performance of FFR $_{\mathrm{CTA}}$ was superior to CTA stenosis for diagnosing ischaemic lesions [15-17]. However, the time span to simulate the transient CFD and calculate FFR $_{\mathrm{CTA}}$ is usually $6 \mathrm{~h}$ [15] or 1-4 $\mathrm{h}$ [17] per examination. Using a reduced-order algorithm, Coenen reported on-site computational FFR $_{\text {CTA }}$ software requiring only 5-10 min to calculate the CFD per patient [18]. However, this method only had a moderate to good correlation $(r=0.59)$. Recently, Zhang et al. employed steady state flow simulation to obtain $\mathrm{FFR}_{\mathrm{SS}}$ and reduced the computational time to $0.5-2 \mathrm{~h}$, together with a good correlation between FFR $_{\mathrm{SS}}$ and invasive FFR $(r=0.843)$ [19]. In addition to the steady state flow simulation applied in Zhang's research, we evaluated the effect of pulsatile flow on FFR $_{\mathrm{CTA}}$ based on a simplified calculation method for the outflow boundary parameters. The aim of this study was to investigate the correlation between the simplified FFR $_{\mathrm{CTA}}$ and invasive FFR in patient-specific coronary arteries, together with the diagnostic performance of the simplified $\mathrm{FFR}_{\mathrm{CTA}}$ in the clinic, and to discuss the feasibility of using this simplified method in identifying ischaemia-related stenosis of CAD. 


\section{Methods}

Populations

This study was approved by the ethical review committee of the First Affiliated Hospital of Jinan University (Guangzhou, Guangdong, China). Since this study is a retrospective study, the informed consent was waived and anonymized data were used for analysis. Coronary CTA performed less than 60 days before scheduled non-emergent ICA and FFR measurement was required for inclusion. Exclusion criteria included individuals who were unable to provide informed consent; complete occlusion of the coronary arteries; significant arrhythmia; non-cardiac illness with life expectancy <2 years; pregnant state; previous coronary intervention or coronary bypass surgery; allergy to iodinated contrast; contraindications to beta-blocking agents, nitroglycerin, or adenosine; and suspected acute coronary syndrome. Eventually, 29 patients in total were included in this study. The average age ranged from 54 to 82 years old (68.1 years old \pm 8.4 years), and the patients were diagnosed with cardiovascular disease between March 15, 2013 and June 23, 2015.

\section{Coronary CTA acquisition and analysis}

Coronary CTA was performed using an MDCT volumetric scanner with 320-detector rows (Aquilion ONE, Toshiba, Otawara, Japan). All the procedures followed the Society of Cardiovascular Computed Tomography guidelines [20]. Oral beta-blockers were administered, targeting a heart rate of $<60$ beats $/ \mathrm{min}$. CCTA data were obtained at both systole and diastole. Experienced radiologists evaluated luminal diameter stenosis in each coronary artery segment using an 18-segment coronary model before ICA [21]. Significant obstruction was defined as luminal stenosis $>50 \%$ in the main coronary arteries.

\section{ICA and FFR measurement}

ICA was performed according to a standard protocol when the severity of stenosis in a major coronary artery was quantified as more than 50\% [22]. Invasive FFR was performed to obtain physiology measurements for clinical indications in significant stenosis. According to the protocol, an FFR pressure wire (PressureWire Aeris/Certus, St. Jude Medical, St. Paul, USA) was positioned distal to the stenosis of interest, at least $3 \mathrm{~cm}$ downstream of the lesion, and then hyperaemia was induced by intravenous infusion of adenosine at $140 \mu \mathrm{g} / \mathrm{kg} / \mathrm{min}$ [23]. FFR was calculated by dividing the mean distal coronary pressure $(\mathrm{mPd})$ by the mean aortic pressure $(\mathrm{mPa})$ during hyperaemia. The FFR was considered diagnostic of ischaemia at a threshold of 0.80 or less [24].

\section{Model establishment}

Patient-specific coronary arterial geometries were reconstructed from 29 sets of CTA image data. By dividing the cross-sectional area of the stenosis by the normal segment proximal to the lesion, 36 lesions were identified as a stenosis by anatomic evaluation. Details of the coronary geometries were determined by the distribution of the contrast agent. Because the coronary lumen was compressed during systole and was unable to be distinguished from the surrounded tissue, the diastole data were used for geometric reconstruction. Vessels were reconstructed offline using Mimics, commercial 3-D 
reconstruction software (Materialise NV, Leuven, Belgium). The mesh of the geometries was generated using a non-structural mesh with tetrahedron elements. The mesh independence test was performed such that different densities of the meshes were generated in one model. The mesh sizes ranged from coarse (approximately 17,100 nodes with 85,600 elements) to fine (approximately 32,800 nodes with 545,820 elements) such that five mesh sizes were generated in total, as shown in Fig. 1. CFD simulation was performed using each mesh, and the maximum velocities from the calculation were considered indexes from which the values were obtained at the same point of the geometry (the centre of the aortic ostium). Convergence of the test was obtained when the difference of the values between two mesh densities was less than $0.1 \%$. The test results indicated that the standard of the finer mesh approach was appropriate for simulations.

\section{CFD configuration and $\mathrm{FFR}_{\mathrm{CTA}}$ computation}

Focusing on the haemodynamics in the coronary artery at the peak flow velocity phase, the flow distribution was assumed to be fully developed in this study. Assumptions were made regarding the simulations that the blood flow was incompressible, laminar and Newtonian; the blood viscosity and density were constant at $0.0035 \mathrm{Pas}$ and $1056 \mathrm{~kg} / \mathrm{m}^{3}$, respectively [25].

The momentum and mass conservation of flow was solved using Navier-Stokes governing equations as follows:

$$
\begin{aligned}
& \rho\left(\frac{\mathrm{du}}{\mathrm{dt}}+\mathrm{u} \cdot \nabla \mathrm{u}\right)=-\nabla p+\mu \nabla^{2} \mathrm{u}+f, \\
& -\nabla \cdot \mathrm{u}=0,
\end{aligned}
$$

where $\rho$ is the density of blood, $\mathrm{u}$ is the velocity field, $\mathrm{p}$ is the pressure, $\mu$ is the viscosity, and $\mathrm{f}$ is the body force per unit volume. All data were obtained while the patients were at rest, and because an external force was not involved, f was assumed to be zero [26].

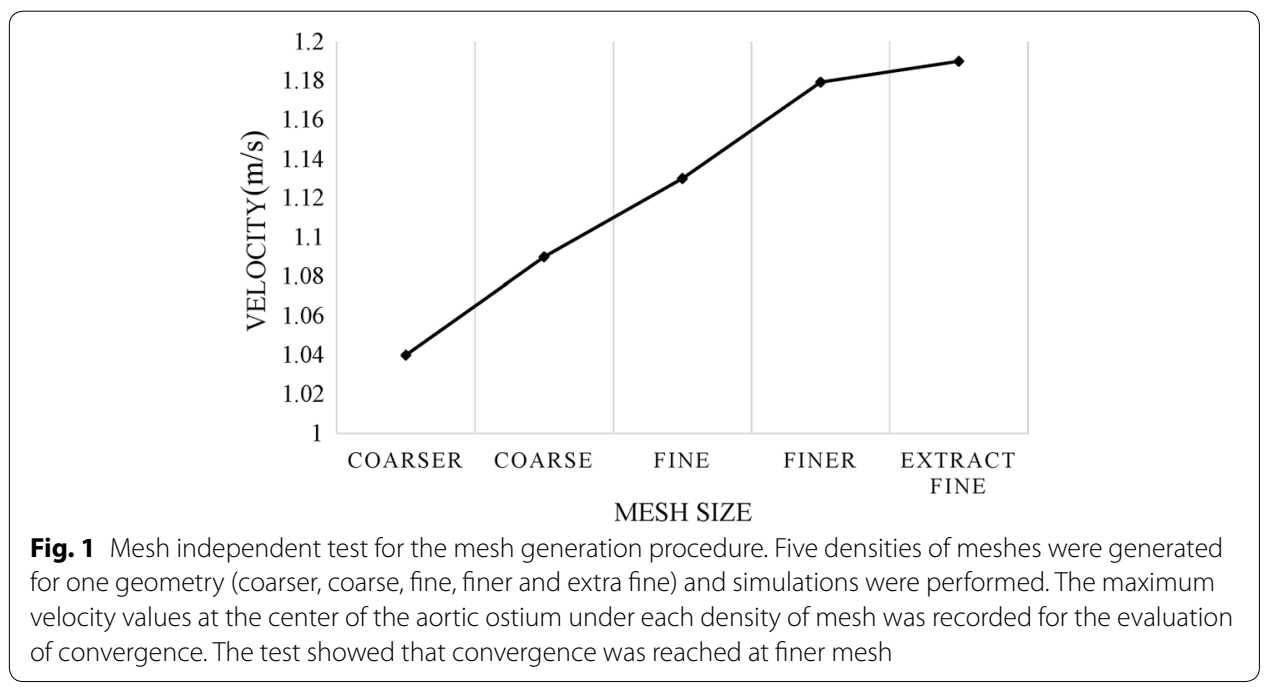


Because pulsatile flow simulation was applied in the present study, the lumped parameter model was implemented for the outflow boundaries. The lumped parameter model (LPM) consisted of resistances and compliances. To achieve the physiological flow condition in arteries, patient-specific parameter values were calculated according to the literature $[19,27]$. In brief, the mean flow rate to the coronary arteries was calculated based on the average physiological condition that the flow to the coronary arteries consumed $4 \%$ of the stroke volume and the ratio of the blood flow between the left and right coronary arteries was 7 to 3 [28]; the relationship between the resistance of each outlet and the total flow in the coronary arteries was determined by the scale of the branch and the mean inlet pressure/flow rate [19]. Then, the resistances of the LPM of each outlet were calculated according to the relationship of the resistances between normal upstream and downstream. The walls of the vessels were assumed to be rigid and to have no-slip boundaries. The normal flow rate of the aorta ostium was implemented at the inflow boundary [27]. For comparison of the accuracy and the effectiveness, the steady state method [19] was also implemented to calculate $\mathrm{FFR}_{\mathrm{SS}}$ in the present study.

Simulations were carried out using COMSOL Multiphysics (COMSOL AB, Stockholm, Sweden), and a multifrontal massively parallel sparse direct solver (MUMPS) was applied to the simulations. $\mathrm{FFR}_{\mathrm{CTA}}$ was calculated by dividing the average pressure at the stenosis by that at the ostium of the coronary artery. The pressure waveform was extracted from the simulations (e.g., Fig. 2), and $\mathrm{FFR}_{\mathrm{CTA}}$ was calculated over one heart cycle period, similar to the measurement procedure during clinical practice. The FFR $_{\text {CTA }}$ based on the simplified method was calculated under the same condition of the computational platform, and the values were extracted directly from the calculations.

\section{Statistical analysis}

Pearson correlation and Bland-Altman plots were performed to investigate the relationships between FFR $_{\text {CTA }}$ and invasive FFR on a per-vessel basis. Invasive FFR was used

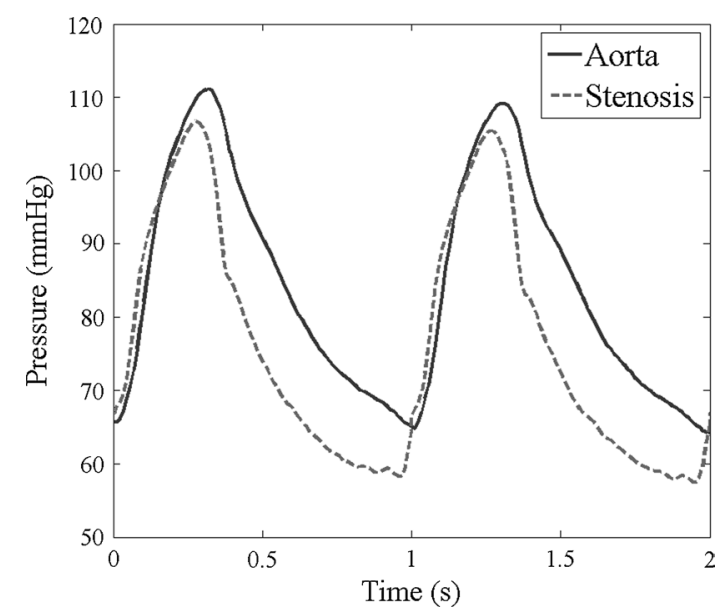

Fig. 2 The pressure waveform at the aorta and the stenosis from the transient simulation. The pressure dropped due to the stenosis compared to the pressure of the aorta. The FFR CTA $_{\text {was calculated as the ratio }}$ dividing the average pressure at the stenosis in one period of heart cycle by the average pressure at the ostium of the coronary artery in the aorta. The FFR $\mathrm{CTA}_{\mathrm{A}}$ value presented in the figure was, for example, 0.88 
as the gold standard (FFR $\leq 0.8$ ) to assess the diagnostic performance of $F_{F R} R_{C T A}$ and the luminal diameter stenosis. A patient was considered positive if any vessel had FFR $\leq 0.8$, and the vessel with the most adverse clinical status was selected to represent a given patient (minimum FFR, minimum FFR $_{\mathrm{CTA}}$ and maximum CCTA stenosis). $\mathrm{FFR}_{\mathrm{CTA}}$ $\leq 0.8$ was used as the threshold to identify the ischaemic lesions in this study, as well as stenosis $>50 \%$. Diagnostic performance on a per-patient and -vessel basis was analyzed, including accuracy, sensitivity, specificity, positive predictive value (PPV), negative predictive value (NPV), positive likelihood ratio (+LR), and negative likelihood ratio ( $-\mathrm{LR})$. The area under the receiver-operator characteristics curve (AUC) was also measured for CCTA stenosis and FFR $\mathrm{CTA}_{\mathrm{A}}$. The AUCs were compared by the DeLong method. A $P$ value less than 0.05 was deemed to be statistically significant. All the analyses were performed on SPSS (version 14, Chicago, IL, USA) and MedCalc Software (MedCalc, Mariakerke, Belgium).

\section{Results}

\section{Baseline characteristics}

The study population included 29 patients who underwent coronary CTA and ICA. Baseline characteristics are listed in Table 1 . The mean interval between the coronary CTA and FFR was 4.3 days (range 0-14 days), with no adverse events or revascularization

Table 1 Baseline characteristics

\begin{tabular}{ll}
\hline Characteristics & Mean \pm SD/n (\%) \\
\hline Age, years & $68.1 \pm 8.4$ \\
Male & $16(55.2 \%)$ \\
Diabeta & $12(41.4 \%)$ \\
Hypertension & $21(72.4 \%)$ \\
Hyperlipidemia & $19(65.5 \%)$ \\
Body mass index, kg/m² & $25 \pm 4$ \\
Current smoker & $13(44.8 \%)$ \\
Cardiovascular history & \\
Previous myocardial infarction & $1(3.4 \%)$ \\
Previous PCl & 0 \\
Previous CABG & 0 \\
Left ventricular ejection fraction, \% & \\
Medications & $60 \pm 7$ \\
Aspirin & \\
Beta-blocker & $15(51.7 \%)$ \\
Nitrate & $23(79.3 \%)$ \\
Statins & $19(65.5 \%)$ \\
ACE inhibitors & $17(58.6 \%)$ \\
Calcium-channel blockers & $26(89.7 \%)$ \\
Clopidogrel & $20(68.9 \%)$ \\
ARBs & $17(58.6 \%)$ \\
Other medication & $4(13.8 \%)$ \\
\hline N 29 patients & $12(41.4 \%)$ \\
\hline
\end{tabular}

$\mathrm{N}=29$ patients

$A C E$ angiotensin-converting enzyme, $A R B$ angiotensin receptor blocker, $C A B G$ coronary artery bypass surgery, $P C I$ percutaneous coronary intervention 
between the tests. FFR and FFR ${ }_{\text {CTA }}$ were evaluated in a total of 36 coronary vessels. The patient and vessel characteristics according to coronary CTA, FFR $\mathrm{CTA}_{\mathrm{CA}}, \mathrm{FFR}_{\mathrm{SS}}$ and FFR are presented in Table 2. On a per-vessel basis, the mean values of measured FFR, FFR $_{\text {CTA }}$ and FFR ${ }_{\text {SS }}$ were $0.81 \pm 0.07,0.78 \pm 0.08$ and $0.78 \pm 0.07$, respectively, and the luminal diameter stenosis evaluated from the CCTA images was $68 \pm 15 \%$. Of the 36 vessels, significant obstruction was observed in $75 \%$ of the vessels, and $47.2 \%$ of the vessels had functionally significant stenosis with FFR $\leq 0.8$.

\section{Analysis of the correlation of $\mathrm{FFR}_{\mathrm{CTA}}$ with FFR and FFR}

As shown in Fig. 3a, good agreement was observed between the simplified FFR $_{\text {CTA }}$ and invasive FFR with a significant difference $(r=0.742, \mathrm{P}<0.001)$. Furthermore, the Bland-Altman plot presented a slight systematic underestimation of FFR $_{\mathrm{CTA}}$ (mean difference 0.03 , standard deviation $0.05, \mathrm{P}=0.001$, Fig. $3 \mathrm{~b}$ ). A negative correlation was also observed between stenosis and invasive FFR $(r=-0.409, \mathrm{P}=0.013$, Fig. 3c). However, a similar correlation of the $\mathrm{FFR}_{\mathrm{SS}}$ obtained from the steady state method with the invasive FFR was found $(\mathrm{r}=0.729, \mathrm{P}<0.001)$, and the Bland-Altman test showed that underestimation was also found in the $\mathrm{FFR}_{\mathrm{SS}}$ obtained by the steady state method (mean difference 0.03 , standard deviation $0.06, \mathrm{P}=0.001$ ). Additionally, the computational efficiency test showed that the computational time spans for the steady state method and simplified pulsatile simulation were $1.2 \pm 0.6 \mathrm{~h}$ and $2.3 \pm 1.2 \mathrm{~h}$, respectively.

\section{Diagnostic performance of $\mathrm{FFR}_{\mathrm{CTA}}, \mathrm{FFR}_{\mathrm{SS}}$ and CCTA stenosis for diagnosis of ischaemia}

The $\mathrm{FFR}_{\mathrm{CTA}}$ obtained for coronary vessels resulted in 16 true positives (44.4\%), 13 true negatives (36.1\%), 6 false positives (16.7\%), and 1 false negative $(2.8 \%)$. On a per-patient basis, $\mathrm{FFR}_{\mathrm{CTA}}$ led to 15 true positives (51.7\%), 8 true negatives (27.6\%), 5 false positives (17.2\%), and 1 false negative (3.4\%). The diagnostic performances of FFR $_{\mathrm{CTA}}, \mathrm{FFR}_{\mathrm{SS}}$ and CCTA stenosis on a per-patient and per-vessel basis are listed in Table 3. Figures 4 and 5 show representative examples of anatomically obstructive CCTA stenosis with and without ischaemia. In Fig. 6, a higher AUC was observed for FFR $\mathrm{CTA}_{\mathrm{A}}$ and $\mathrm{FFR}_{\mathrm{SS}}$ compared with CCTA stenosis on a per-vessel basis $(0.93 / 0.88 / 0.75)$, as well as on a per-patient basis $(0.90 / 0.84 / 0.71)$.

Table 2 Patient and vessel characteristics according to coronary CTA, FFR CTA, $_{\text {, FFR }}$ and FFR

\begin{tabular}{lc}
\hline Characteristics & $\mathbf{n}(\%)$ \\
\hline Patients with coronary CTA maximum stenosis $>50 \%$ & $23(79.3)$ \\
Patients with minimum FFR $\leq 0.8$ & $16(55.2)$ \\
Patients with minimum FFR & $20(68.9)$ \\
Patients $\leq 0.8$ & $22(75.9)$ \\
Vessels with minimum $F F R_{S S} \leq 0.8$ & $27(75.0)$ \\
Vessels with FFR $\leq 0.8$ & $17(47.2)$ \\
Vessels with FFR & $22(61.1)$ \\
Vessels $\leq 0.8$ & $24(66.7)$ \\
\hline $\mathrm{N}=29$ patients $F F R_{S S} \leq 0.8$ & \\
$F F R$ fractional flow reserve, $36 R_{C T A}$ fractional flow reserve calculated with the pulsatile flow simulation basing coronary & \\
computed tomography angiography datasets, $F F R_{S S}$ fractional flow reserve calculated with the steady state method
\end{tabular}



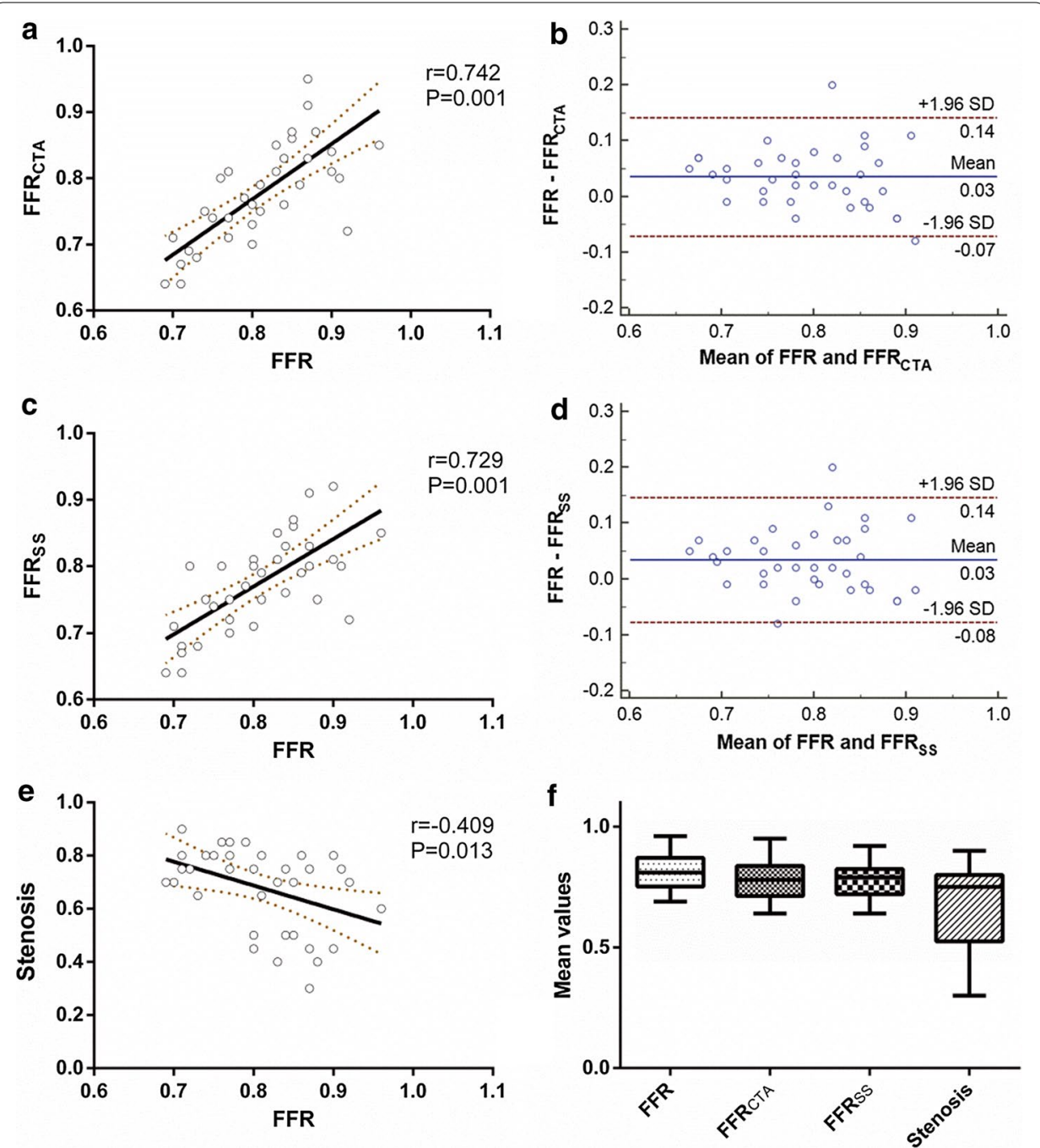

Fig. 3 Comparison among CCTA stenosis, FFR $\mathrm{CTA}_{\mathrm{A}}, F F R_{S \mathrm{~S}}$ and invasive FFR on a per-vessel basis. a Pearson correlation between FFR $\mathrm{CTA}_{\mathrm{A}}$ and invasive FFR, $r$ was 0.742 with significant difference $(P=0.001)$. b Bland-Altman plots of FFR CTA $_{\text {and }}$ invasive FFR, mean difference 0.03 , standard deviation 0.05. c Pearson correlation between $F_{S F}$ and invasive FFR, $r$ was 0.729 with significant difference $(P=0.001)$. $\mathbf{d}$ Bland-Altman plots of FFR $R_{S S}$ and invasive FFR, mean difference 0.03 , standard deviation 0.06 . e Pearson correlation between stenosis and invasive FFR, $r$ was -0.409 with significant difference $(P=0.013)$. f Mean vlaue of FFR, FFR $\mathrm{CTA}, F F R_{S S}$ and stenosis

\section{Discussion}

At present, the fractional flow reserve (FFR) has been regarded as the gold standard in the assessment of haemodynamic characteristics for coronary stenosis [5], and it is recommended for making clinical treatment decisions before coronary revascularization [6]. FFR is applied to guide management in less than $10 \%$ of PCI cases, especially in developing countries, because of the high cost and invasive procedure [8]. In recent years, with the development of computational fluid dynamics (CFD), it is possible to calculate the coronary flow and pressure from anatomic imaging data [14]. FFR $\mathrm{CTA}_{\mathrm{A}}$ has emerged as a new non-invasive method and has been investigated worldwide. 


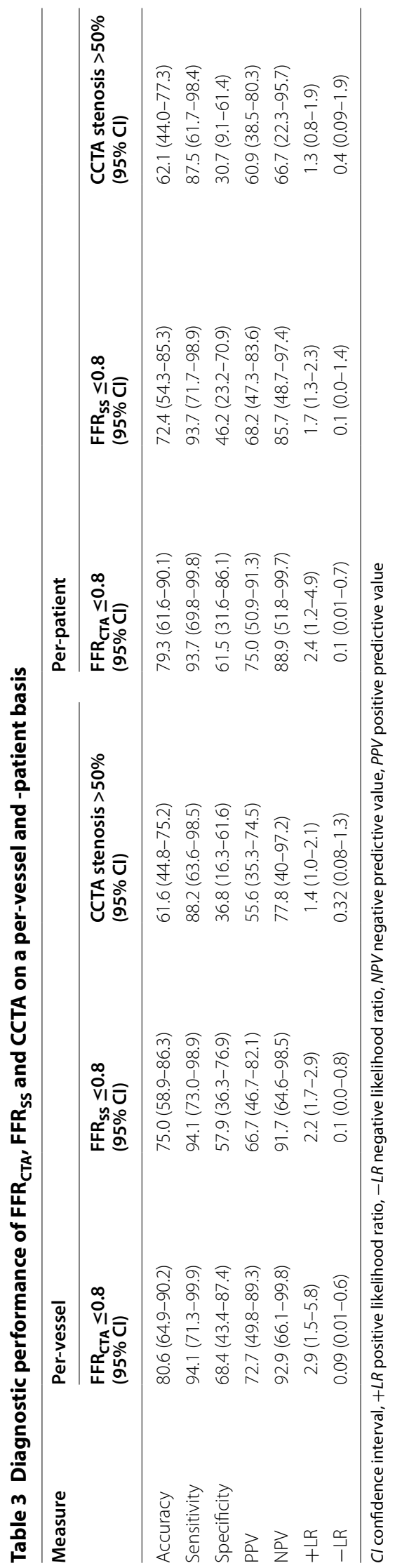




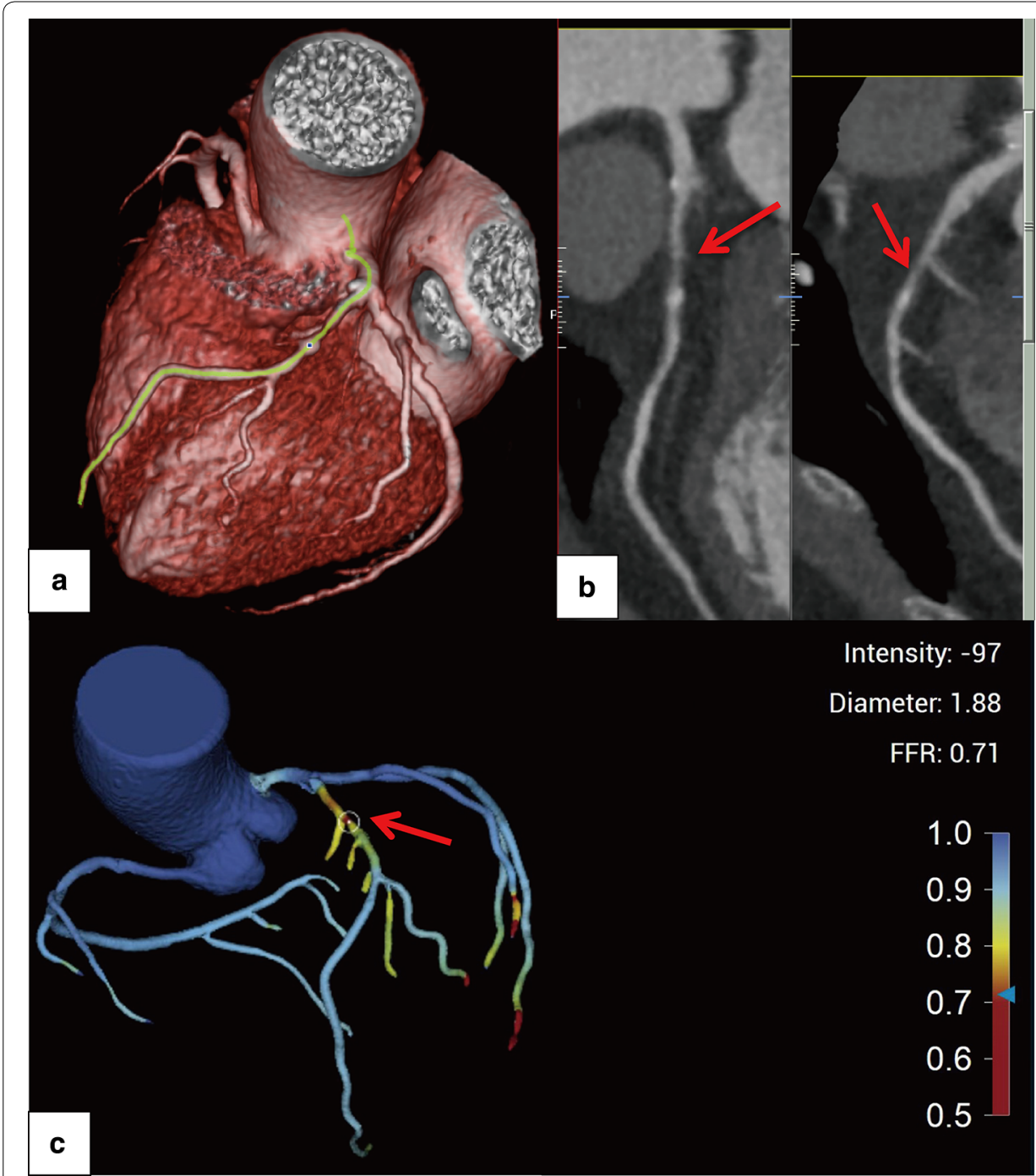

Fig. 4 Volume-rendered image (a) and multiplanar reformat (b) of CCTA and FFR $\mathrm{C}_{\mathrm{CTA}}$ (c) of the left anterior descending artery (LAD). CCTA demonstrates stenosis (80\% lumen reduction) of the proximal-portion of LAD

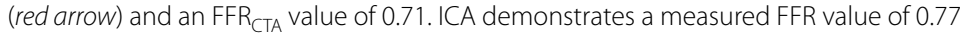

In this study, $\mathrm{FFR}_{\mathrm{CTA}}$ was calculated by implementing pulsatile flow simulation with novel parameter estimation. By comparing the correlation of $\mathrm{FFR}_{\mathrm{CTA}}$ and $\mathrm{FFR}_{\mathrm{SS}}$ with the invasive FFR in patient-specific coronary arteries, the accuracy of the FFR $_{\mathrm{CTA}}$ calculated by using pulsatile flow simulation in the present study is slightly higher than thas using steady state simulations [19]. The area under the curve (AUC) was used to evaluate the diagnostic performance per-vessel and per-patient. Our result showed that FFR $\mathrm{CTA}_{\mathrm{A}}$ has higher diagnostic performance and a larger AUC than CCTA stenosis alone; the result was equivalent to Norgaard and Zhang's research except for a decreased specificity. However, the overall diagnostic performance in the present study was lower compared to previous studies $[18,19]$; this may mainly be related to the calcification of the stenosis in the present study that is commonly found in stenosis in the clinic. The artefacts from calcification decrease the apparent lumen and lead to narrow coronary segmentations, 


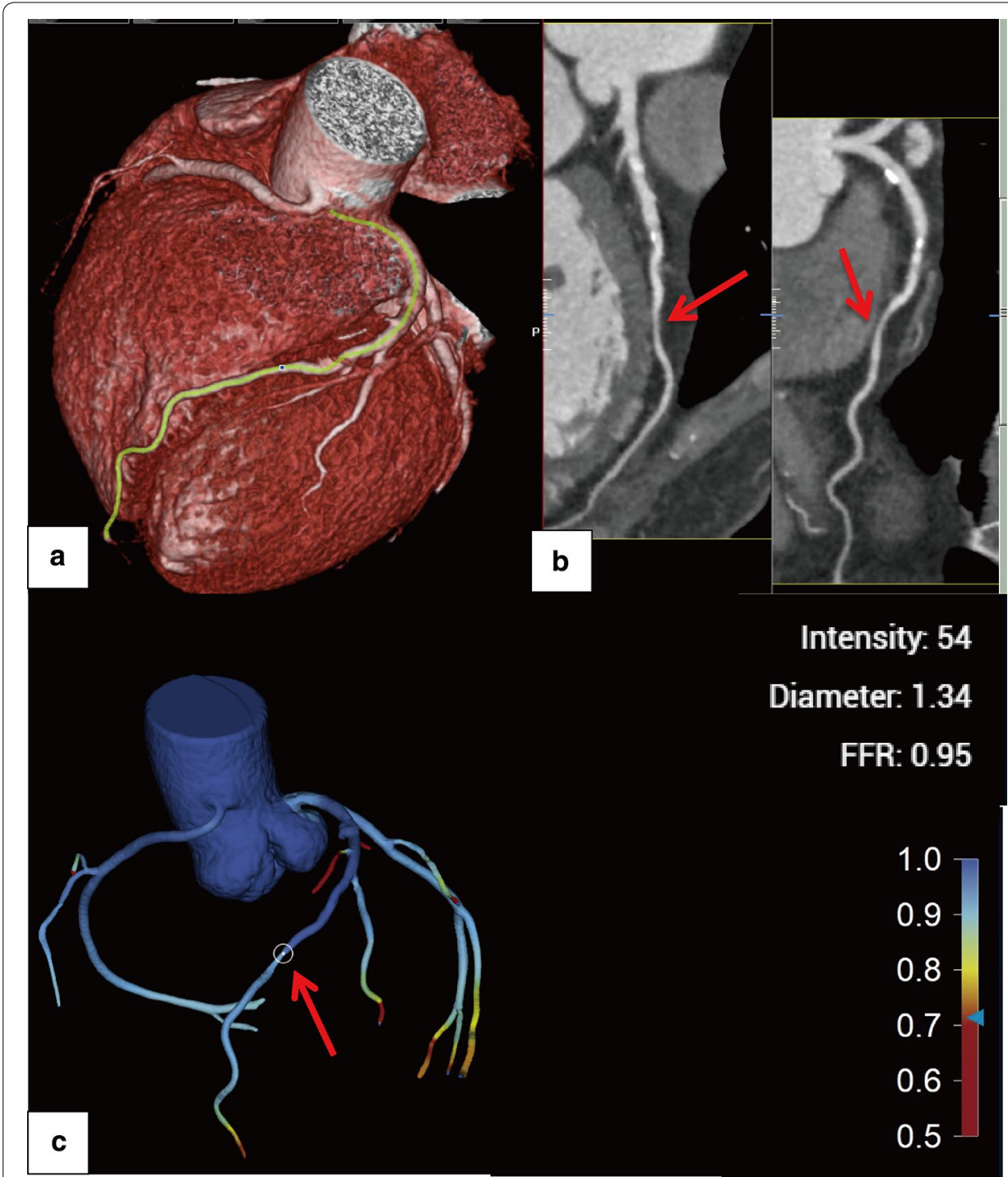

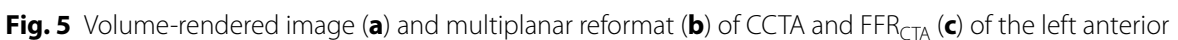
descending artery (LAD). CCTA demonstrates stenosis (75\% lumen reduction) of the mid-portion of LAD (red

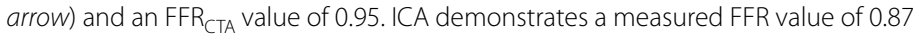

resulting in lower FFR $\mathrm{CTA}_{\mathrm{A}}$ values and an increased proportion of false positive cases [29]. Overall, the results presented in our study showed that it is possible to obtain FFR $_{\mathrm{CTA}}$ based on the simplified method with pulsatile flow simulation and a novel boundary condition within a reduced computational time.

The calculation of FFR derived from CT imaging data represents an alternative approach in the assessment of haemodynamic characteristics for coronary stenosis. In clinical practice, the severity of stenosis shows a poor relationship with ischaemia [30], as shown in Fig. 3e. Especially in patients with stenosis in the intermediate range (30$70 \%$ ), it is hard to judge whether the severity of the stenosis would lead to ischaemia without an invasive FFR measurement through an expensive coronary pressure wire. The application of $\mathrm{FFR}_{\mathrm{CTA}}$ is conducive to reducing the false-positive cases caused by 

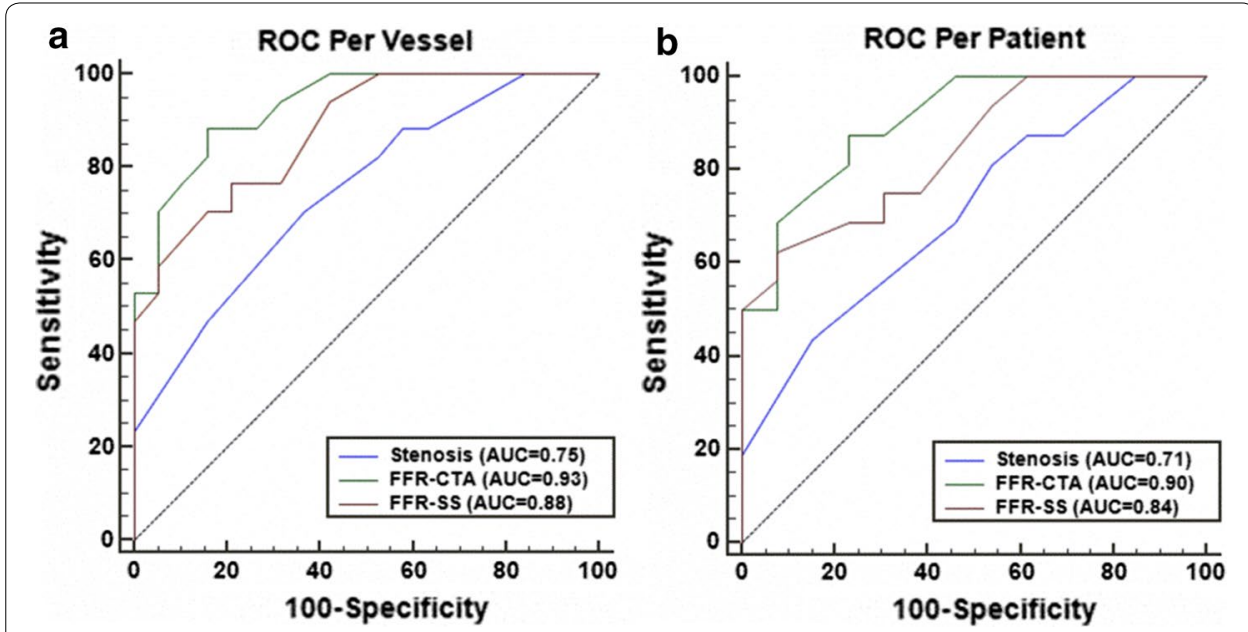

Fig. 6 Area under the receiver-operating characteristic curve (AUC) of FFR ${ }_{C T A}, F F R_{S S}$ and CCTA stenosis for discriminating ischemia on $\mathbf{a}$ a per-vessel and $\mathbf{b}$ per-patient basis separately

coronary CT angiography findings and cutting down the need for a second diagnostic examination. Despite an incremental diagnostic performance of $\mathrm{FFR}_{\mathrm{CTA}}$ having been reported by several clinical trials, its application is still limited because of the demanding process. Comparing the pulsatile flow simulation that was used in the DISCOVERFLOW [15], DeFACTO [16] and NXT [17] trials, the simplified method applied in the present study can reduce the simulation time span significantly and maintain a superior diagnostic discrimination characteristic. However, the value of the simplified method was debatable in that the transient fluid dynamic analysis is still an effective tool, especially for the complex distribution of multiple stenoses. In addition, the high quality of the CT image and the consistent CTA protocol are also important to improved diagnostic performance of $\mathrm{FFR}_{\mathrm{CTA}}$ [17]. With the use of an MDCT volumetric scanner with 320 -detector rows, finer detector elements of $0.5 \mathrm{~mm}$ compared with many other CT scanners can be achieved in this study.

In clinical practice, several tests have been established as non-invasive methods to provide functional diagnostic information, such as single photon emission computed tomography (SPECT), coronary magnetic resonance imaging (cMRI) or stress echocardiography. These methods can provide useful information on patient prognosis, and thus they have been recommended for evaluating patients with symptoms in the guidelines [31]. Several studies have investigated the ability of these tests to identify ischaemia. In Jogiya's research, the sensitivity, specificity, and diagnostic accuracy of cMRI for the detection of significant CAD were 91,90 , and $91 \%$, respectively [32]. In another study of early dipyridamole stress, for myocardial SPECT to detect residual stenosis, the sensitivity and specificity of SPECT to detect the functionally and morphologically significant residual stenosis were 92 and 31\% and were 83 and 29\%, respectively [33]. In Jung's study of dobutamine stress echocardiography, a sensitivity of $48 \%$ and a specificity of $73 \%$ were reported $[34,35]$. However, these methods do not visualize the stenotic coronary arteries and cannot provide haemodynamic information of the individual coronary lesions compared with CCTA and FFR $\mathrm{CTA}_{\mathrm{A}}$. With continually rising healthcare costs, more attention 
is focused on the cost effectiveness of procedures. The present study supported that, as an alternate diagnostic parameter, the $\mathrm{FFR}_{\mathrm{CTA}}$ calculated by this simplified method has the potential to be an available gatekeeper to ICA and revascularization compared with the invasive FFR measurement and could reduce healthcare costs for patients suspected of having CAD at the same time. In addition, $\mathrm{FFR}_{\mathrm{CTA}}$ can also be used to predict the haemodynamic changes resulting from percutaneous coronary intervention or coronary artery bypass graft. Likewise, the method established in our study has the potential to be generalized to peripheral vascular disease, such as carotid, renal and cerebral vascular stenosis.

Several limitations exist in the present study. There are several outliers observed in Fig. 3a, b. The reasons may lie in the following aspects: (1) the limited resolution in the small vessel of the CCTA could result in the deviation between reconstructed geometries and actual anatomy, contributing bias to the calculations; (2) the timespan for the pulsatile flow simulation was longer than that for the steady state simulations, so a more efficient algorithm is required to advance the clinical implementation of FFR $_{\text {CTA; }}$; (3) because the coronary arteries are fixed on the heart wall, the deformation of the vessel walls caused in the end-diastolic phase is not included; (4) the small size of samples obtained in the present study prevented us from further analyzing the stenosis in the intermediate range (30-70\%), which showed the poorest relationship with ischaemia in the clinic; and (5) because patients with acute coronary syndromes or previous coronary intervention or bypass surgery were not included in the present study, whether this method can be applied to these patients still needs to be studied.

\section{Conclusion}

In this study, a simplified method algorithm was employed to calculate $\mathrm{FFR}_{\mathrm{CTA}}$; we observed good correlation and an acceptable mean difference between FFR $_{\mathrm{CTA}}$ and invasive FFR, as well as a better diagnostic performance of FFR $_{\text {CTA }}$ in diagnosing ischaemia-causing stenosis in the clinic. By implementing this new boundary condition, the simplified FFR $_{\mathrm{CTA}}$ calculated with pulsatile flow has the potential to be an alternate and accurate diagnostic parameter in the assessment of the haemodynamic characteristics for coronary stenosis.

\footnotetext{
Abbreviations

CAD: coronary artery disease; ICA: invasive coronary angiography; FFR: fractional flow reserve; FAME: fractional flow reserve versus angiography for multivessel evaluation; $\mathrm{PCl}$ : percutaneous coronary intervention; CCTA: coronary computed tomographic angiography; CFD: computational fluid dynamics; LPM: lumped parameter model; MUMPS: multifrontal massively parallel sparse direct solver; PPV: positive predictive value; NPV: negative predictive value; +LR: positive likelihood ratio; -LR: negative likelihood ratio; AUC: area under the receiver-operator characteristics Curve; SPECT: single photon emission computed tomography; CMRI: coronary magnetic resonance imaging.
}

\section{Authors' contributions}

CZS, DZ, TZ, and LPL conceptualized the study; CZS, DZ, and TZ contributed to the methodology; XL and HYZ contributed to software; CZS, DZ, and TZ validated the results; CZS, DZ, and TZ contributed to formal analysis; CZS, DZ, TZ contributed to investigation; CZS, DZ, TZ, and LPL contributed to resources; CZS, DZ, and TZ contributed to data curation; $C Z S, D Z, T Z$, and $X L$ wrote the original draft of the manuscript; CZS, DZ, TZ, and HYZ contributed to review and editing of the manuscript; CZS, DZ, TZ, and XL contributed to visualization; LPL, HYZ, and KLC contributed to supervision; LPL, $\mathrm{XL}$, and $\mathrm{HYZ}$ contributed to project administration; $\mathrm{LPL}$ and $\mathrm{HYZ}$ contributed to funding acquisition. All authors read and approved the final manuscript.

Author details

${ }^{1}$ Medical Imaging Center, The First Affiliated Hospital, Jinan University, 613 Huangpu W Ave, Tianhe District, Guangzhou 510630, Guangdong Province, China. ${ }^{2}$ Research and Development Department, Shenzhen Keya Medical 
Technology, Co., Ltd., Longgang District, Shenzhen 518116, Guangdong Province, China. ${ }^{3}$ Shenzhen Institutes of Advanced Technology, Chinese Academy of Sciences, 1068 Xueyuan Ave. Xili University Town, Nanshan District, Shenzhen 518055, Guangdong Province, China.

\section{Acknowledgements}

Not applicable.

\section{Competing interests}

The authors declare that they have no competing interests.

\section{Availability of data and materials}

The datasets analyzed during the current study are not publicly available because of the further analysis of the datasets in our research but are available from the corresponding author upon reasonable request.

\section{Declaration and verification}

All the authors state that the work described has not been published previously, that it is not under consideration for publication elsewhere, that its publication is approved by all authors and tacitly or explicitly by the responsible authorities where the work was carried out, and that, if accepted, it will not be published elsewhere in the same form, in English or in any other language, including electronically without the written consent of the copyright holder.

\section{Ethics approval and consent to participate}

The study was approved by the ethical review committee of the First Affiliated Hospital of Jinan University (Guangzhou, Guangdong, China) (Study Number: 2016025) and was conducted in accordance with the Helsinki Declaration. Since this study is a retrospective study, the requirement for informed consent was waived and anonymized data were used for analysis.

\section{Funding}

This study received funding from the Collaborative Innovation Program of Hong Kong and Guangdong Province, the Guangdong Science and Technique Department (Nos. 2016A050503032, 2015B010125005), the National Natural Science Foundation of China (No. 61427807), Shenzhen Innovation Funding (SGLH20150213143207911, JCYJ20151030151431727), and the National Key Research and Development Program of China (2016YFC1300302, 2016YFC1301700)

Received: 18 December 2016 Accepted: 17 March 2017

Published online: 14 April 2017

\section{References}

1. He J, et al. Major causes of death among men and women in China. N Engl J Med. 2005;353(11):1124-34.

2. Shaw $L$, et al. Optimal medical therapy with or without percutaneous coronary intervention to reduce ischemic burden: results from the Clinical Outcomes Utilizing Revascularization and Aggressive Drug Evaluation (COURAGE) trial nuclear substudy. Circulation. 2008;117(10):1283-91.

3. Lucas FL, et al. Diagnostic-therapeutic cascade revisited: coronary angiography, coronary artery bypass graft surgery, and percutaneous coronary intervention in the modern era. Circulation. 2008;118(25):2797-802.

4. van Rosendael AR, et al. Relation between quantitative coronary CTA and myocardial ischemia by adenosine stress myocardial CT perfusion. J Nucl Cardiol. 2016;1-10. doi:10.1007/s12350-016-0393-7.

5. Tonino PA, et al. Angiographic versus functional severity of coronary artery stenoses in the FAME study fractional flow reserve versus angiography in multivessel evaluation. J Am Coll Cardiol. 2010;55(25):2816-21.

6. Pijls $\mathrm{NH}$, et al. Measurement of fractional flow reserve to assess the functional severity of coronary-artery stenoses. $\mathrm{N}$ Engl J Med. 1996;334(26):1703-8.

7. De Bruyne $\mathrm{B}$, et al. Fractional flow reserve-guided $\mathrm{PCl}$ versus medical therapy in stable coronary disease. N Engl J Med. 2012;367(11):991-1001.

8. Fearon WF, et al. Economic evaluation of fractional flow reserve-guided percutaneous coronary intervention in patients with multivessel disease. Circulation. 2010;122(24):2545-50.

9. Morris PD, et al. Virtual fractional flow reserve from coronary angiography: modeling the significance of coronary lesions: results from the VIRTU-1 (VIRTUal fractional flow reserve from coronary angiography) study. JACC CardiovasC Interv. 2013;6(2):149-57

10. Siebert $U$, et al. Improving the quality of percutaneous revascularisation in patients with multivessel disease in Australia: cost-effectiveness, public health implications, and budget impact of FFR-guided PCI. Heart Lung Circ. 2014;23(6):527-33.

11. Trivedi $\mathrm{S}$, et al. Measurement of myocardial fractional flow reserve is a cost-effective way to identify coronary artery lesions of indeterminate severity that warrant revascularisation. Heart Lung Circ. 2005;14(4):239-41.

12. Achenbach S, Daniel WG. Noninvasive coronary angiography_an acceptable alternative? N Engl J Med. 2001;345(26):1909-10.

13. Budoff MJ, et al. Diagnostic performance of 64-multidetector row coronary computed tomographic angiography for evaluation of coronary artery stenosis in individuals without known coronary artery disease: results from the prospective multicenter ACCURACY (assessment by coronary computed tomographic angiography of individuals undergoing invasive coronary angiography) trial. J Am Coll Cardiol. 2008;52(21):1724-32.

14. Min JK, Shaw $\amalg$, Berman DS. The present state of coronary computed tomography angiography a process in evolution. J Am Coll Cardiol. 2010;55(10):957-65. 
15. Kim HJ, et al. Patient-specific modeling of blood flow and pressure in human coronary arteries. Ann Biomed Eng. 2010;38(10):3195-209.

16. Koo BK, et al. Diagnosis of ischemia-causing coronary stenoses by noninvasive fractional flow reserve computed from coronary computed tomographic angiograms. Results from the prospective multicenter DISCOVER-FLOW (diagnosis of ischemia-causing stenoses obtained via noninvasive fractional flow reserve) study. J Am Coll Cardiol. 2011;58(19):1989-97.

17. Min JK, et al. Diagnostic accuracy of fractional flow reserve from anatomic CT angiography. JAMA. 2012;308(12):1237-45.

18. Norgaard BL, et al. Diagnostic performance of noninvasive fractional flow reserve derived from coronary computed tomography angiography in suspected coronary artery disease: the NXT trial (analysis of coronary blood flow using CT angiography: next steps). J Am Coll Cardiol. 2014;63(12):1145-55.

19. Coenen $A$, et al. Fractional flow reserve computed from noninvasive $C T$ angiography data: diagnostic performance of an on-site clinician-operated computational fluid dynamics algorithm. Radiology. 2015;274(3):674-83.

20. Zhang JM, et al. Simplified models of non-invasive fractional flow reserve based on CT images. PLoS ONE. 2016;11(5):e0153070.

21. Abbara S, et al. SCCT guidelines for performance of coronary computed tomographic angiography: a report of the Society of Cardiovascular Computed Tomography Guidelines Committee. J Cardiovasc Comput Tomogr. 2009;3(3):190-204.

22. Raff GL, et al. SCCT guidelines for the interpretation and reporting of coronary computed tomographic angiography. J Cardiovasc Comput Tomogr. 2009;3(2):122-36.

23. Naidu SS, et al. Clinical expert consensus statement on best practices in the cardiac catheterization laboratory: Society for Cardiovascular Angiography and Interventions. Catheter Cardiovasc Interv. 2012;80(3):456-64.

24. De Bruyne B, et al. Intracoronary and intravenous adenosine 5'-triphosphate, adenosine, papaverine, and contrast medium to assess fractional flow reserve in humans. Circulation. 2003;107(14):1877-83.

25. Tonino PA, et al. Fractional flow reserve versus angiography for guiding percutaneous coronary intervention. $N$ Engl J Med. 2009;360(3):213-24.

26. Liu B. The influences of stenosis on the downstream flow pattern in curved arteries. Med Eng Phys. 2007;29(8):868-76.

27. Iguchi T, et al. Impact of lesion length on functional significance in intermediate coronary lesions. Clin Cardiol. 2013;36(3):172-7.

28. Li-Na P, et al. A quantitative study of the effect of blood viscosity and bulk modulus of plaque compositions on the vulnerability of an atherosclerotic plaque using a 3D fluid-structure interaction model. In: Proceedings of 2012 IEEEEMBS international conference on biomedical and health informatics. 2012.

29. Opie LH. Heart physiology: from cell to circulation. Philadelphia: Lippincott Williams \& Wilkins; 2004.

30. Coenen A, et al. Coronary CT angiography derived fractional flow reserve: methodology and evaluation of a point of care algorithm. J Cardiovasc Comput Tomogr. 2016;10(2):105-13.

31. Meijboom WB, et al. Comprehensive assessment of coronary artery stenoses: computed tomography coronary angiography versus conventional coronary angiography and correlation with fractional flow reserve in patients with stable angina. J Am Coll Cardiol. 2008;52(8):636-43.

32. Task Force M, et al. 2013 ESC guidelines on the management of stable coronary artery disease: the Task Force on the management of stable coronary artery disease of the European Society of Cardiology. Eur Heart J. 2013;34(38):2949-3003.

33. Jogiya $R$, et al. Validation of dynamic 3-dimensional whole heart magnetic resonance myocardial perfusion imaging against fractional flow reserve for the detection of significant coronary artery disease. J Am Coll Cardiol. 2012;60(8):756-65.

34. Seo JK, et al. Early dipyridamole stress myocardial SPECT to detect residual stenosis of infarct related artery: comparison with coronary angiography and fractional flow reserve. Korean J Intern Med. 2002;17(1):7-13.

35. Jung PH, et al. Effect of contrast application on interpretability and diagnostic value of dobutamine stress echocardiography in patients with intermediate coronary lesions: comparison with myocardial fractional flow reserve. Eur Heart J. 2008;29(20):2536-43.

\section{Submit your next manuscript to BioMed Central and we will help you at every step:}

- We accept pre-submission inquiries

- Our selector tool helps you to find the most relevant journal

- We provide round the clock customer support

- Convenient online submission

- Thorough peer review

- Inclusion in PubMed and all major indexing services

- Maximum visibility for your research

Submit your manuscript at www.biomedcentral.com/submit 